service being superseded by testing by polymerase chain reaction (PCR)-testing of nose/throat swabs, for which only 1 was sent for the entire cohort.

Conclusion While BC collection was frequent, compliance with local guidance was less than desired. Sampling to rule out atypical pathogens in CAP appeared to be low and, occasionally, inappropriate. Although positive detection rates were low, further work appears to be indicated to optimise patient outcomes in CAP by increasing awareness amongst clinicians of tests available and ensure appropriate sampling for atypical pathogen screening.

\section{P213 CXR FOLLOW-UP AFTER COMMUNITY ACQUIRED PNEUMONIA (CAP): OUTCOMES OF ADHERENCE TO GUIDELINES}

${ }^{1} \mathrm{P}$ Eaton, ${ }^{2} \mathrm{HJ}$ Curtis. ${ }^{1}$ Newcastle University, Newcastle Upon Tyne, UK; ${ }^{2}$ Queen Elizabeth Hospital, Gateshead, UK

\subsection{6/thoraxjnl-2015-207770.349}

Introduction and objectives BTS Community Acquired pneumonia (CAP) guidelines require a chest radiograph (CXR) 6 weeks after discharge in high risk patients. This is the hospital team's responsibility. Malignancy is reported in $1.7 \%$ of patients.

We wanted to assess how this was organised across our trust and the outcomes, aiming to improve organisation of the service.

Methods Patients were identified via retrospective review of local NIV/COPD and critical care ICNARC databases. Xrays and reports were reviewed and eRecords interrogated for appointments, follow-up CXR and outcomes.

Results 102 patients were identified between December 2013 to January 2015, (mean age 69 and 56 female patients). Only 54 patients had the follow up CXR. 16 patients did not need local follow-up for a variety of reasons: co-morbidities; current malignant diagnosis; CXR clear pre-discharge or usual residence outside geographical area. 7 patients did not attend the organised follow up appointment. Ultimately, 25 patients had no follow up plans made.

The majority of follow-up CXR were organised by the hospital team $(\mathrm{n}=53)$ compared to discharge correspondence instructions to GP to request a CXR $(\mathrm{n}=14)$. The hospital requested CXR were significantly more likely to be undertaken, with $88 \%$ completed versus $57 \%$ from requests to GP (p $<0.05$ Chi-square).

The majority of CXRs showed resolution of changes $(n=38$ / 54), 3 patients had lung or pleural malignancy diagnosed, 2 patients are having on-going nodule follow-up and 1 patient had atypical mycobacterium diagnosed. 10 patients to date have incomplete resolution of their changes. These end-points were after 62 CXRs, $7 \mathrm{Ct}$ scans, 2 bronchoscopies and 1 pleural biopsy.

Conclusion There is room for improvement within our trust to improve this parameter for CAP patients. This would be best fulfilled by automatic request at time of discharge follow CAP.

The follow up clearly requires increasing amounts of work and administration. How does the NHS keep up with guideline requirements and clinical outcomes in the ageing population?

The finding of $5.5 \%(3 / 54)$ new malignancy shows the importance of follow-up and is higher than published reports.

\section{P214 ARRHYTHMIAS IN PNEUMONIA: A REVIEW OF INCIDENCE, OUTCOMES AND MANAGEMENT}

DR Cox. University Hospital Aintree, Liverpool, UK

\subsection{6/thoraxjnl-2015-207770.350}

Background Community-acquired pneumonia (CAP) has high mortality, from 5 to $18.3 \% .^{1}$ Arrhythmias are a recognised significant complication. Growing evidence associates this treatable complication with increases in mortality. No review has summated data on mortality or ways to improve outcome.

Aims This review aimed to define the extent of the problem, collate and appraise evidence regarding outcomes and management, and identify gaps in understanding.

Methods Narrative review using a systematic protocol. Medline, ProQuest, Web of Science were searched for papers reporting adults with CAP complicated by arrhythmia. 382 articles were assessed and excluded based on title (348), abstract (27) and full text (6), leaving 11 . Review of bibliographies added 3, totalling 14. These were appraised and coded, with Newcastle-Ottawa scores assigned.

Results Three reviews and 11 primary studies were included: 10 Cohorts (4 prospective, 6 retrospective) and one case series. One meta-analysis of cardiac events identified a pooled incidence of $4.7 \%$ for CAP inpatients developing arrhythmia. ${ }^{2} \mathrm{~N}-\mathrm{O}$ scores ranged from 5 to 9, Median 7.5. Outcomes reported: Incidence; 30 and 90 day mortality; Re-hospitalisation; predisposing factors. Only one paper commented on treatment.

Discussion There is high quality evidence of a link between CAP and arrhythmogenesis. Data linking it to mortality suggest a strong association with worse outcome. This review was limited by its single reviewer. Some evidence was limited by retrospective study designs and biassed populations. The strengths of this review lie in its reproducible systematic methods and clear outlining of gaps in our understanding of this phenomenon, particularly regarding best management.

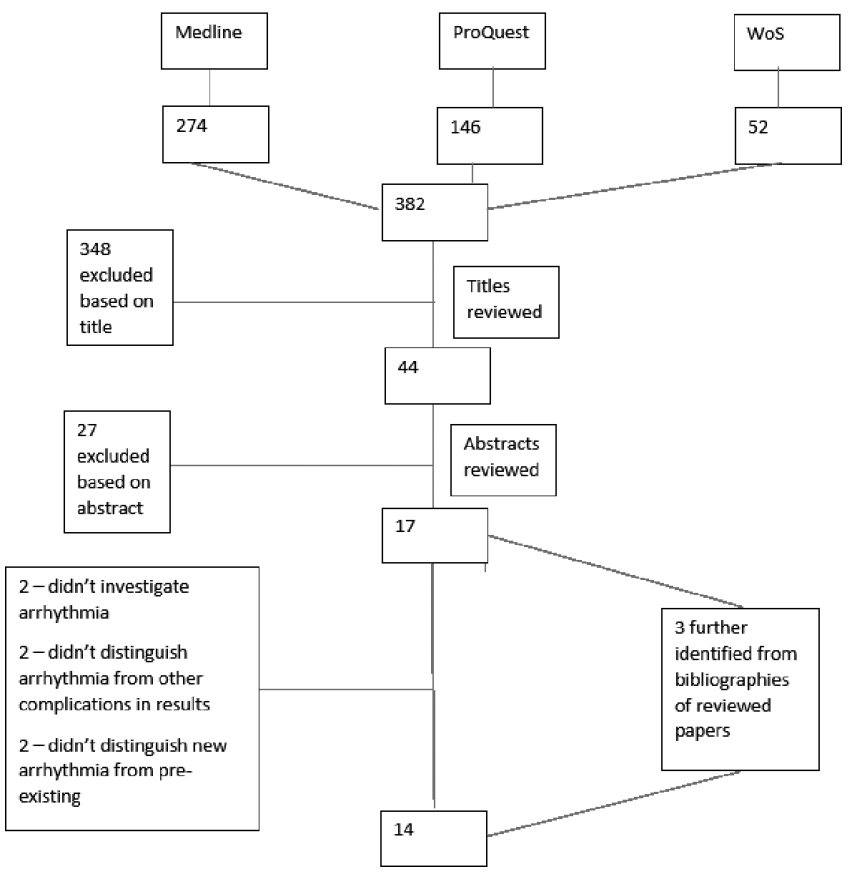

Abstract P214 Figure 1 
Conclusion New arrhythmia complicating CAP is a recognised phenomenon that carries morbidity and mortality. Notably, no research has been reported on how best to manage this complication - reflected by the guidelines for the respective diseases in isolation. The next step is to look at how this complication is managed and identify the best approach to improve patient outcomes.

\section{REFERENCES}

1 Lim WS, Woodhead M. BTS adult CAP audit 2009/10. Thorax 2011:66(6): $548-549$

2 Corrales-Medina VF, et al. Cardiac complications in patients with communityacquired pneumonia: a systematic review and meta-analysis of observational studies. PLoS Med. 2011:8(6):779

\section{Epidemiology in lung disease}

\section{P215 THE EPIDEMIOLOGY OF PNEUMOTHORAX IN ENGLAND (1968-2011)}

RJ Hallifax, R Goldacre, NM Rahman, M Goldacre. University of Oxford, Oxford, UK

\subsection{6/thoraxjnl-2015-207770.351}

Introduction and objectives Spontaneous Pneumothorax (SP) is a common pathology. Incidence rates are quoted as 16-24 and 1.2-6 per 100,000 cases per annum for males and females respectively, based on two studies in single centres (45 years ago, USA; 30 years ago, Sweden) and 4 -year periods of national data in UK (1991-4) and France (2008-2011).

The aim of this study is to determine the incidence and recurrence of spontaneous pneumothorax in a larger dataset in England.

Methods An all-England Hospital Episode Statistics (HES) dataset from 1968-2011 was used to determine the incidence of Spontaneous Pneumothorax using International Classification of Diseases codes as the main diagnosis in a hospital admission. A record-linked HES dataset (only available from 1999-2011) was used to distinguish between Primary and Secondary Spontaneous Pneumothorax (PSP and SSP) and to determine the risk of a second pneumothorax within specified time intervals. SSP was defined as the patient having a diagnosis of a chronic lung disease (e.g. COPD, emphysema, lung malignancy, asthma, bronchiectasis, sarcoidosis) made at any time covered by the linked data.

Results and discussion From 1968-2011, in a population of 50 million, there were a total of 246,534 episodes of spontaneous pneumothorax (no data for 1986-89). In 1999-2011, the average annual incidence was 9.1 per 100,000 males and 3.2 per 100,000 females for PSP; 11.9 and 4.7 for SSP; and 21.0 and 7.9 for SP overall. The incidence of SP appears to be increasing (Figure 1): it was 12.5 (95\% confidence interval 12.2-12.8) in 1999 and $13.6(13.3-13.9)$ in 2011. It is unclear whether this reflects a true rise in new cases, better reporting or increasing recurrence rates.

The overall risk of recurrence is $13.5 \%$ within 1 year $(18.7 \%$ within 5 years). Recurrence is more common in SSP than PSP at 1 year $(16.1 \%$ vs $10.6 \%)$ and 5 years $(21.2 \%$ vs $14.7 \%)$.

Conclusions This is the largest epidemiological study of pneumothorax to date. These data only cover hospitalised pneumothorax, and therefore may be a conservative estimate of the true burden of disease. Pneumothorax appears to be increasing in incidence.

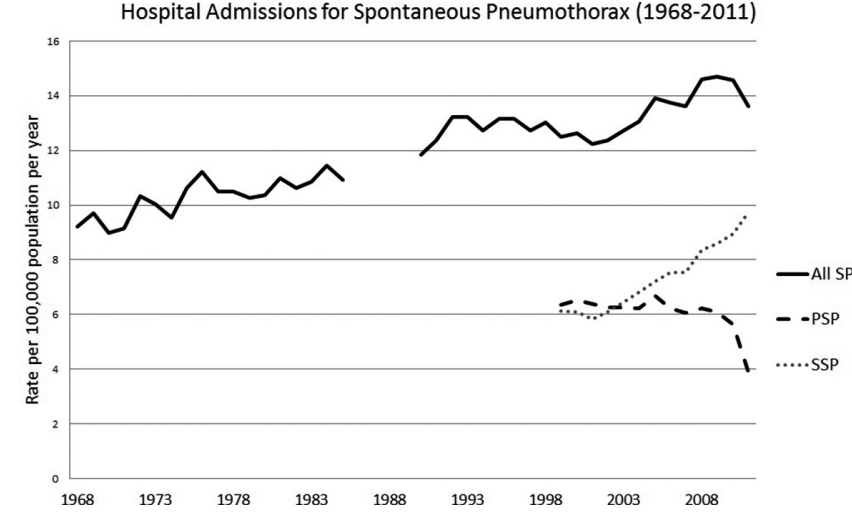

Abstract P215 Figure 1

\section{P216 DURATION OF TOTAL AND EXCLUSIVE BREASTFEEDING, TIMING OF SOLID FOOD INTRODUCTION AND RISK OF ALLERGIC DISEASES: A SYSTEMATIC REVIEW AND META-ANALYSIS}

${ }^{1} V$ Garcia-Larsen, ${ }^{2} \mathrm{D}$ lerodiakonou, ${ }^{3} \mathrm{~J}$ Leonardi-Bee, ${ }^{4} \mathrm{~T}$ Reeves, ${ }^{2} \mathrm{~J}$ Chivinge, ${ }^{2} \mathrm{Z}$ Robinson, ${ }^{2} \mathrm{~K}$ Jarrold, ${ }^{2} \mathrm{~N}$ Geoghegan, ${ }^{2} \mathrm{E}$ Andreou, ${ }^{5} \mathrm{~N}$ Tagiyeva-Milne, ${ }^{6} \mathrm{U}$ Nurmatov, ${ }^{1} \mathrm{~S}$ Cunha, ${ }^{2}$ RJ Boyle. 'Respiratory Epidemiology, Occupational Medicine and Public Health Group, NHLI, Imperial College London, London, UK; ${ }^{2}$ Department of Paediatrics, Imperial College London, London, UK; ${ }^{3}$ Division of Epidemiology and Public Health, School of Medicine, University of Nottingham, Nottingham, UK; ${ }^{4}$ Central Library, Imperial College London, London, UK; ${ }^{5}$ University of Aberdeen, Aberdeen, UK; ${ }^{6}$ Centre for Population Health Sciences, University of Edinburgh, Edinburgh, UK

\subsection{6/thoraxjnl-2015-207770.352}

Background Allergic diseases are the leading causes of chronic illness in children and young adults in the UK.

Aim To undertake a comprehensive review of the evidence on the effect of breastfeeding (BF) duration and timing of solid food introduction (SFI), on the risk of wheeze, atopic dermatitis, rhino-conjunctivitis, food allergy, allergic sensitisation and measures of lung function or bronchial hyper-responsiveness.

Methods We carried out a systematic review following the PRISMA guidelines (International Prospective Register of Systematic Reviews [PROSPERO] CRD42013003802). We included intervention, cohort, case-control and cross-sectional studies. Following literature searches (July 2013), study eligibility, data extraction and risk of bias assessments were conducted independently by two investigators. Random effects meta-analyses were used to pool results. Five levels of comparison of total or exclusive $\mathrm{BF}$ duration were used to assess disease risk in children at age $0-4$ yrs, $5-15$ yrs or $15+$ yrs: 'never vs ever',' $\geq 1-2$ months vs. $<1-2$ months', ' $\geq 3-4$ months vs. $<3-4$ months', ' $\geq 5-7$ months vs. $<5-7$ months', and ' $\geq 8-12$ months vs. $<8-$ 12 months'. Exclusive BF (EBF; BF without formula or solid food supplementation) was categorised as ' $\geq 0-2$ months vs. $<0$ 2 months', ' $\geq 3-4$ months vs. $<3-4$ months' and ' $\geq 5+$ months vs. $<5+$ months', and SFI as ' $\geq 3-4$ months vs. $<3-4$ months'. Publication bias was assessed using Egger's asymmetry test.

Results Of 16,289 identified studies, 564 met the inclusion criteria and were eligible for analysis. We found reduced risk of wheezing in children aged 5-14 yrs with longer BF or EBF duration, which was dose-dependent, but there was evidence of publication bias ( $\mathrm{BF}$ and odds of recurrent wheezing $\mathrm{P}=0.007$ ). Similar results were found for recurrent wheeze at age 5-14 yrs but not in other ages. Measures of lung function were also increased with increased BF or EBF duration. We found no 